


\title{
The challenge of brain complexity: A brief discussion about a fractal intermittency-based approach
}

\author{
Paolo Paradisi ${ }^{1,2}$, Marco Righi ${ }^{1}$, and Umberto Barcaro ${ }^{3}$ \\ ${ }^{1}$ Istituto di Scienza e Tecnologie dell'Informazione “A. Faedo" (ISTI-CNR), Via G. Moruzzi 1, 56124 Pisa, Italy \\ ${ }^{2}$ Basque Center of Applied Mathematics (BCAM), Alameda Mazarredo 14, 48009 Bilbao, Bizkaia, Spain \\ ${ }^{3}$ Dipartimento di Informatica, Universitá di Pisa, Largo B. Pontecorvo 3, 56127 Pisa \\ paolo.paradisi@cnrit
}

Keywords: Biomedical signal processing, ElectroEncephaloGram, brain events, fractal intermittency, threshold analysis, pattern recognition, complex systems

\begin{abstract}
In the last years, the complexity paradigm is gaining momentum in many research fields where large multidimensional datasets are made available by the advancements in instrumental technology. A complex system is a multi-component system with a large number of units characterized by cooperative behavior and, consequently, emergence of well-defined self-organized structures, such as communities in a complex network. The self-organizing behavior of the brain neural network is probably the most important prototype of complexity and is studied by means of physiological signals such as the ElectroEncephaloGram (EEG). Physiological signals are typically intermittent, i.e., display non-smooth rapid variations or crucial events (e.g., cusps or abrupt jumps) that occur randomly in time, or whose frequency changes randomly. In this work, we introduce a complexity-based approach to the analysis and modeling of physiological data that is focused on the characterization of intermittent events. Recent findings about self-similar or fractal intermittency in human EEG are reviewed. The definition of brain event is a crucial aspect of this approach that is discussed in the last part of the paper, where we also propose and discuss a first version of a general-purpose event detection algorithm for EEG signals.
\end{abstract}

\section{INTRODUCTION}

A crucial focus in Physiological Computing is the development of human-computer interaction devices whose applications span from the monitoring of the health state of a patient, with an associated decision support system including diagnosis and prognosis, to the application of specific therapies, (e.g., administration of stimuli).

In this general framework, the so-called complexity approach (Solé and Bascompte, 2006; Sornette, 2006), focused on the analysis and modeling of emerging self-organization in multi-component systems and complex networks, is nowadays gaining momentum in the field of biomedical signal processing. In order to extract useful information from large clinical datasets, storing many different physiological data and signals, algorithms for the reduction of data complexity are needed to derive reliable diagnostic indices. Then, a great interest is focused in defining, developing and testing statistical indices that can enclose the minimal information required to characterize physiological signals in a both efficient and reli- able way. This is of relevance in clinical activities involving, e.g., disorders of consciousness (DOC) (De Biase et al., 2014; Monti et al., 2010; Fingelkurts et al., 2013; Fingelkurts et al., 2014; Laureys and Tononi, 2009; Tononi, 2008) or attention deficit hyperactivity disorders (ADHD) (Robbie et al., 2016).

However, such indices are useful if they are able to describe the key features of the signals and if these features can be exploited by physicians in their clinical activity, e.g., in the evaluation of a medical condition or disease (diagnosis); in foretelling the course of a disease (prognosis); in the consequent choice of the proper therapy (decision making). Interestingly, the possibility of applying complex stimuli with fractal features as innovative therapeutic strategies is attracting the attention of many researchers (Zueva, 2015; Hägerhäll et al., 2015; Spehar et al., 2015).

In this work we introduce and discuss an approach to the processing of ElectroEncephaloGram (EEG) signals that is based on the observation that, in many complex systems, such as the human physiology, the dynamics trigger crucial events, each one associated 
with the emergence or decay of self-organized structures in the system (Allegrini et al., 2009; Allegrini et al., 2010a; Allegrini et al., 2010b; Allegrini et al., 2011; Paradisi et al., 2013; Allegrini et al., 2013; Allegrini et al., 2015; Beggs and Plenz, 2003; Plenz and Thiagarjan, 2007; Fraiman et al., 2009; Chialvo, 2010; Grigolini and Chialvo, 2013).

The paper is organized as follows. In Section 2 we introduce the fractal intermittency description of complex systems and we discuss a measure of complexity and a reliable method to estimate it. In Section 3 we review some results about the application of fractal intermittency to brain dynamics, introducing the basic concept of crucial event in the brain. In Section 4 we discuss a proposal for a general-purpose algorithm for event detection in human EEG and in Section 5 we draw some conclusions.

\section{The paradigm of fractal intermittency in complexity}

Even if a general definition of complex system is not universally accepted in the scientific community, some features are recognized to be ubiquitous in the presence of complexity (Paradisi et al., 2015c; Paradisi et al., 2015b; Solé and Bascompte, 2006; Sornette, 2006). Without demanding completeness, we give a brief list of the most important features:

- A complex system is composed of many parts, i.e., degrees of freedom: many individuals, particles, units or, in general, many sub-systems that are embedded in a network of strong nonlinear interactions.

- The behavior of a multi-component system can be considered complex if the nonlinear dynamics is cooperative, thus giving rise to self-organized or coherent states.

- Non-reducibility: self-organized states have temporal and spatial scales that are hardly obtained as a simple (linear) function of an external forcing or by means of linear coarse graining procedures (e.g., average or weighted sum over single components).

- Self-organized states display long-range space and/or time correlations (slow power-law decay). The correlation exponents are an important example of emergent properties that can be used as synthetic indicators of the cooperative dynamics in the complex system.

In order to introduce the approach discussed in this paper, it is worth noting that the above list of features is not complete. In fact, the following general observations are also in order:
- Self-organized states are usually metastable states. They are in fact characterized by relatively long life-times and by fast transition events among two successive coherent states.

- The sequence of fast transition events among selforganized states is described by a intermittent birth-death point process of self-organization (i.e., coherence): $\left\{t_{n}\right\} ; t_{n+1}>t_{n} ; t_{0}=0 ; n=0,1,2, \ldots$, being $t_{n}$ the occurrence times of the $n$-th event. Even if the states are in a far-from-equilibrium condition, the overall dynamics are stationary (on average) if there are no external perturbations.

- The life-times of coherent structures are defined by the time intervals between two successive transition events: $\tau_{n}=t_{n}-t_{n-1} ; n=1,2, \ldots$ In complex systems these times, here denoted as Waiting Times (WTs), are random variables whose statistical distribution has a inverse power-law tail: $\psi(\tau) \sim 1 / \tau^{\mu}$ (Allegrini et al., 2009; Allegrini et al., 2010a; Allegrini et al., 2010b; Allegrini et al., 2011; Paradisi et al., 2013; Allegrini et al., 2013; Allegrini et al., 2015).

- The fast transitions among metastable states are typically associated with a fast memory drop in the dynamics, so that each self-organized state is often independent from each other, as such as the transition events. This is denoted as renewal condition and the sequence of transition events is a renewal point process (Cox, 1962; Paradisi and Allegrini, 2015; Paradisi et al., 2015a; Paradisi et al., 2012a; Paradisi et al., 2012b; Paradisi et al., 2009b; Akin et al., 2006; Paradisi et al., 2008; Akin et al., 2009; Bianco et al., 2007; Paradisi et al., 2009a) In this case the WTs $\tau_{n}$ are mutually independent random variables. Conversely, in the time interval $\left(t_{n}, t_{n+1}\right)$ between two events, i.e., in correspondence of a self-organized state, the dynamics are strongly correlated.

The inverse power-law in the WT distribution is the manifestation of a self-similar behavior in the cooperative dynamics of the complex system. This powerlaw behavior in the WT distribution is a crucial emergent property, characterizing the capacity of the complex system to trigger self-organization. This condition is denoted as fractal intermittency (Paradisi et al., 2012b; Paradisi et al., 2013; Allegrini et al., 2013; Paradisi et al., 2015b; Paradisi and Allegrini, 2015). This complex behavior is also known as Temporal Complexity (Grigolini, 2015; Beig et al., 2015; Turalska et al., 2011; Grigolini and Chialvo, 2013), a term that was introduced to underline the difference of the intermittency-based approach to complexity, focused on the study of the temporal structure of self- 
organization, with the more extensively investigated approach associated with the estimation of topological and spatial indicators of complexity (e.g., the degree distribution in a complex network, the avalanche size distribution) (Beggs and Plenz, 2003; Plenz and Thiagarjan, 2007; Fraiman et al., 2009; Chialvo, 2010; Grigolini and Chialvo, 2013).

In summary, when a system is characterized by fractal intermittency, the essential dynamics are an alternation of metastable states, with strong coherence and long life-times, and transition (intermittent) events that occur randomly in time, develop in very short time, can be considered instantaneous and are associated with a fast memory drop. In this condition, the experimental observation of long-range (power-law) correlations is strictly connected to the inverse powerlaw decay in the statistical distribution of the random life-times (Allegrini et al., 2009).

\subsection{Intermittency-based scaling analysis as a signature of complexity}

The emergence of intermittency per se is not a signature of complexity. In fact, let us consider a intermittent system with many units whose dynamics is independent from each other. An example of this condition is given by neural cultures in vitro with connections that are chemically inhibited. The absence of interactions involves the absence of cooperation and, thus, of self-organized structures. Independently from the single neuron intermittent dynamics, the overall behavior of the network is described by a renewal Poisson process (Cox, 1962), which involves an exponential WT distribution. This simple observation implies that Poisson intermittency in a multi-dimensional system without external perturbations is associated with absence of self-organization and, thus, it can be taken as a non-complexity reference condition. Then, a necessary condition for complex self-organization in intermittent systems is the emergence of crucial events with non-Poisson statistics.

The emergence of non-Poisson statistics by itself is not a signature of complexity but, as observed above, a inverse power-law tail in the WT distribution is often observed in complex systems. As a consequence, the power exponent $\mu$ can be exploited as a signature of complex behavior and it is denoted as complexity index. This intermittency-based complexity measure is very powerful and represents a robust way of quantifying the amount of complexity in a system, being the value of $\mu$ typically associated with the ability of the system of triggering complex self-organized structures.

\subsection{Diffusion scaling and the EDDiS method}

The complexity index $\mu$ is formally defined by the asymptotic behavior of the WT distribution. However, due to secondary pseudo-events associated with the presence of noise in the signals and/or with the unavoidable limitations of the event detection algorithm, the direct estimation of $\mu$ is often not possible as a clear power-law decay is not seen in the hystogram (Allegrini et al., 2010a; Paradisi et al., 2012a; Paradisi et al., 2012b; Paradisi and Allegrini, 2015) (Allegrini et al., 2010a) proved that, when a intermittent process with complexity index $\mu$ is superposed to noisy events, the WT distribution can display an apparent exponent $\mu^{\prime}$ that is completely different from the underlying real $\mu$. For this reason, (Allegrini et al., 2009) exploited an algorithm for the indirect estimation of $\mu$. In this method, denoted as analysis of Event-Driven Diffusion Scaling (EDDiS), three different diffusion processes driven by the same sequence of event occurrence times $\left\{t_{n}\right\}$ are firstly built. Then, some scaling exponents of the event-driven diffusion processes are evaluated by applying well-known tools of statistical data analysis: the Detrended Fluctuation Analysis (DFA) for the second moment scaling $H:\left\langle X^{2}(t)\right\rangle \sim t^{2 H}$ (Peng et al., 1994); and the Diffusion Entropy (DE) for the self-similarity exponent $\Delta: P(x, t)=F\left(x / t^{\Delta}\right) / t^{\Delta}$ (see Ref. (Paradisi and Allegrini, 2015) and references therein).

The EDDis method exploits a combination of some theoretical results about the relationships between $\mu$ and the exponents $H$ and $\mu$ that are well-known in literature by many years (for a short review see Ref. (Paradisi and Allegrini, 2015) and references therein). Theoretically, it should possible to get 6 different estimates of $\mu$, but sometimes not all the estimates are reliable due to the presence of noise (Allegrini et al., 2010a; Paradisi and Allegrini, 2015). In any case, at least 2 values of $\mu$ can be usually obtained. Being the relationships $H=H(\mu)$ and $\Delta=\Delta(\mu)$ based on the renewal assumption, the self-consistency of the $\mu$ values is used as a test for the renewal assumption and, in the positive case, this also gives a robust estimation of $\mu$ (Paradisi et al., 2012b; Paradisi and Allegrini, 2015). The EDDiS method is used to estimate the complexity index $\mu$ in the presence of noisy pseudo-events. However, the scaling exponents $H$ and/or $\Delta$ can be also used directly to characterize the complexity of the system. Actually, when the renewal condition is satisfied, using the diffusion scaling or the power-law exponent $\mu$ is totally equivalent. 


\section{Complexity and fractal intermittency in the brain}

Brain dynamics is probably the most important example of complex self-organization with a great variety of quantitatively and qualitatively different behaviors, also spanning over several time scales. The efficiency of brain information processing is strictly associated with the metastability of the highly non-stationary neural assemblies continuously forming and decaying, usually in response to external and internal stimuli in order to maintain the homeostatic equilibrium. In this section we introduce the fractal intermittency approach in brain research and we briefly discuss some interesting findings about brain complexity.

\subsection{Transition events in brain dynamics}

Fingelkurts and Fingelkurts proposed a conceptual model of the brain, the Brain Operation Architectonics, that could explain the self-organizing and multi-scale behavior of brain dynamics (Fingelkurts and Fingelkurts, 2001; Fingelkurts et al., 2008; Fingelkurts et al., 2010; Fingelkurts and Fingelkurts, 2015; Fingelkurts et al., 2013). According to this scenario, a neural assembly is a group of neurons for which coordinated activity persists over substantial time intervals and underlies basic operations of information processing during brain functioning. Thus, the neural assemblies are the metastable, self-organized, states introduced in Section 2.

From this brain theory, a general approach to the signal processing of EEG data was derived (Kaplan et al., 2005). In this paper EEG signals are recognized to be strongly non-stationary, reflecting the switching among different neural assemblies. During the lifetime of neural assemblies, the EEG signals should display periods of quasi-stationarity, while rapid changes can be seen in the EEG traces in correspondence of the emergence and decay of the neural assemblies themselves. These rapid changes are called Rapid Transition Processes (RTPs) and mark the passages between two quasi-stationary periods and, as said above, are interpreted as the birth or death of metastable neural assemblies (Kaplan et al., 2005).

\subsection{Deep sleep, wakefulness and REM: towards an index for consciousness}

RTPs are a prototype of complex transition events in the brain and RTP definition was exploited to apply the EDDiS method introduced above. This allowed to discover the presence of fractal intermittency in the brain functioning (Allegrini et al., 2009; Allegrini et al., 2010a; Allegrini et al., 2010b; Allegrini et al., 2011; Paradisi et al., 2013; Allegrini et al., 2013; Allegrini et al., 2015). The interesting range of the complexity index is: $1<\mu<3$. (Allegrini et al., 2009) found an approximate value $\mu=2.1$ for the brain basal condition (wakefulness with closed eyes). The interesting result is that this value remain quite stable among different subjects. More interestingly, $\mu$ was proven to be associated with the degree of integration/segregation of the brain dynamics and, thus, with consciousness (Paradisi et al., 2013; Allegrini et al., 2013; Allegrini et al., 2015). This result was obtained by applying the EDDiS method to EEG data recorded during sleep condition, including about 30 minutes before the sleep onset. This allowed to estimate the diffusion scaling $H$, whose values was found to be in agreement with the integrated states of consciousness and the segregated states of unconscious deep sleep. These results indicate that intermittency-based complexity measures could be good candidates as indicators of the consciousness state of a patient with brain injury.

\section{Towards a general-purpose event detection algorithm}

In our opinion, even if the global RTP events extracted from EEG signals are good candidates to characterize the state of consciousness of a subject, the application of the fractal intermittency approach for general purposes would require an extension in the definition of brain events. In fact, EEG signals are strongly non-stationary and display large variability at several time scales and this variability is not only quantitative but also qualitative with very different waveforms and grapho-elements, also depending on the experimental condition. It is then desiderable to extend the RTP definition of brain event to a multi-modal definition, thus allowing to have different kind of events that can be used individually or jointly depending on the particular application and scope. For example, we expect that a kind of brain events is suitable to establish some pathological condition, while another kind is not.

The starting point here proposed is based on the standard approach based on splitting the EEG signal into the classical frequency bands. For each band, some basic events are extracted, so that we can define at least one event typology for each band. In our opinion, this would allow to implement a first kernel of event detection algorithms that can be improved and upgraded in future developments. Further, in brain research each frequency band is often the starting point for the building of neural correlates. This could allow for a easier link between the technical aspects of the signal processing procedure used to extract the events and the neuro-physiological interpretation of these same events. Then, the intermittency-based complexity index can be also extended to a vector of 
complexity measures, whose elements can be selected and/or coupled in different ways depending of the particular application and/or pathology in order to get a reliable statistical index.

The approach here introduced is essentially an extension of an already existing algorithm that was originally developed to detect the high activity epochs within each frequency band by means of a thresholding technique (see, e.g., (Navona et al., 2002; Barcaro et al., 2004; Magrini et al., 2015; Righi et al., 2008)) In the intermittency-based complexity approach the focus is on the transition events from high $(+)$ to low (-) states (epochs) and vice versa. The event detection algorithm is applied to EEG signals after a preprocessing, essentially consisting of the usual notch filter at $50 \mathrm{~Hz}$ and a successive artifact removal. In the following we assume that the EEG signal was already pre-processed and artifacts were removed.

Then, the event detection algorithm works as follows:

(1) The frequency bands of the EEG signal are computed. The following band ranges are usually considered: (a) $\delta$ band $(0.5-4 \mathrm{~Hz})$; (b) $\theta$ band $(4-8$ $\mathrm{Hz})$; (c) $\alpha$ band $(8-12 \mathrm{~Hz})$; (d) $\sigma$ band $(12-16$ $\mathrm{Hz})$; (e) $\beta$ band $(16-35 \mathrm{~Hz})$; (f) $\gamma$ band $(35-64$ $\mathrm{Hz}$ ).

(2) For each frequency band, the absolute value, i.e., the component amplitude is considered. Then, two moving-window time averages are computed at different time scales, short and long, that are typically chosen as 2 and $64 \mathrm{sec}$, respectively.

(3) Calculation of non-dimensional descriptors for each frequency band: (short-time average - longtime average)/long-time average [denoted as $A=$ $A(t)$ in Fig. 1].

(4) Identification of epochs and transition events between epochs for a given band. In Fig. 1 we sketch an example with 6 different kinds of events, all obtained by considering the crossings through two different thresholds: L (Low) and $\mathrm{H}$ (High). Typical values are $\mathrm{L}=0$ and $\mathrm{H}=1$. In the example of Fig. 1, the epoch typologies are: (i) $L_{ \pm}$: epochs with descriptor above $(+)$or below (-) the threshold L; (ii) $H_{ \pm}$: same as before for the threshold $\mathrm{H}$; (iii) $C_{+}$: epochs $L_{+}$containing at least one epoch $H_{+}$; (iv) $\mathrm{C}_{-}$(complementary to the epochs $C_{+}$. Similarly, the crossing times corresponding to these epochs define the events of the kind $L_{ \pm}, H_{ \pm}$and $C_{ \pm}$.

(5) Storing in a database (spatio-temporal event maps) and feature extraction (e.g., number of events per time unit for each band and EEG trace).

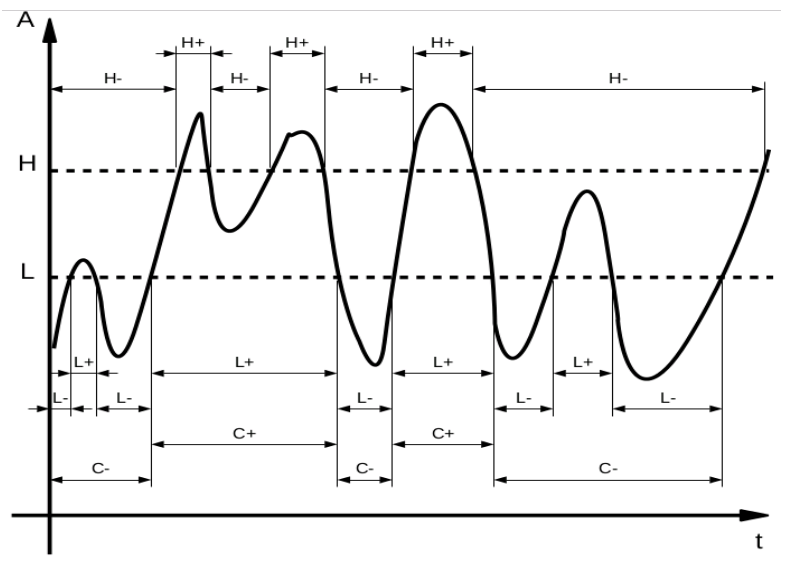

Figure 1: Identification of epochs and transition events. The curve $A=A(t)$ is the descriptor defined in item (3) for a given frequency band, $\mathrm{L} / \mathrm{H}$ is the low/high threshold.

\section{CONCLUSIONS}

In this work we have discussed the complexity paradigm in the context of the analysis and modeling of physiological signals and, in particular, the fractal intermittency approach applied to the signal processing of EEG recordings. We argued that the operational definition of the events and, consequently, the specific algorithm of event detection can play a crucial role in the evaluation of EEG complexity associated with fractal intermittency. For this reason, we discussed a proposal to extend the definition of RTP events in order to have a set of different kind of brain events that could be used for different applications and to build a general-purpose algorithm for event detection and, thus, for the estimation intermittencybased complexity measures.

\section{REFERENCES}

Akin, O., Paradisi, P., and Grigolini, P. (2006). Physica A, 371:157-170.

Akin, O. C., Paradisi, P., and Grigolini, P. (2009). Journal of Statistical Mechanics: Theory and Experiment, page P01013.

Allegrini, P., Menicucci, D., Bedini, R., Fronzoni, L., Gemignani, A., Grigolini, P., West, B., and Paradisi, P. (2009). Physical Review E, 80:061914.

Allegrini, P., Menicucci, D., Bedini, R., Gemignani, A., and Paradisi, P. (2010a). Physical Review E, 82:015103.

Allegrini, P., Paradisi, P., Menicucci, D., Bedini, R., Gemignani, A., and Fronzoni, L. (2011). Journal of Physics: Conference Series, 306:012027.

Allegrini, P., Paradisi, P., Menicucci, D., and Gemignani, A. (2010b). Frontiers in Physiology, 1:128.

Allegrini, P., Paradisi, P., Menicucci, D., Laurino, M., Bedini, R., Piarulli, A., and Gemignani, A. (2013). Chaos Solitons \& Fractals, 55:32-43. 
Allegrini, P., Paradisi, P., Menicucci, D., Laurino, M., Piarulli, A., and Gemignani, A. (2015). Physical Review E, 92:032808.

Barcaro, U., Bonanni, E., Maestri, M., Murri, L., Parrino, L., and Terzano, M. (2004). Sleep Medicine, 5:567576.

Beggs, J. and Plenz, D. (2003). Journal of Neuroscience, 23:11167-11177.

Beig, M., Svenkeson, A., Bologna, M., West, B., and Grigolini, P. (2015). Physical Review E, 91:012907.

Bianco, S., Grigolini, P., and Paradisi, P. (2007). Chemical Physics Letters, 438(4-6):336-340.

Chialvo, D. (2010). Nature Physics, 6:744-750.

Cox, D. (1962). Renewal Theory. Methuen \& Co. Ltd., London.

De Biase, S., Gigli, G., and et al. (2014). Sleep Medicine, 15:393400.

Fingelkurts, A. and Fingelkurts, A. (2001). Brain Mind, 2:261-296.

Fingelkurts, A. and Fingelkurts, A. (2015). In Sakkalis, V., editor, Modern Electroencephalographic Assessment Techniques: Theory and Applications, Neuromethods Book Series, vol. 91, pages 1-59. Springer, New York.

Fingelkurts, A., Fingelkurts, A., Bagnato, S., and andG. Galardi, C. B. (2014). Frontiers Human Neuroscience, 8(402). doi: 10.3389/fnhum.2014.00402.

Fingelkurts, A., Fingelkurts, A., and Neves, C. (2008). The Open Neuroimaging Journal, 2:73-93.

Fingelkurts, A., Fingelkurts, A., and Neves, C. (2010). Physics of Life Reviews, 7:195-249.

Fingelkurts, A., Fingelkurts, A., and Neves, C. (2013). Chaos, Solitons \& Fractals, 55:13-31.

Fraiman, D., Balenzuela, P., Foss, J., and Chialvo, D. (2009). Physical Review E, 79:061922.

Grigolini, P. (2015). Chaos, Solitons \& Fractals, 81, Part B:575-588.

Grigolini, P. and Chialvo, D., editors (2013). volume 55 of Chaos, Solitons \& Fractals. Elsevier, Amsterdam. Special Issue, Pages 1-120.

Hägerhäll, C., Laike, T., Küller, M., Marcheschi, E., Boydston, C., and Taylor, R. (2015). Nonlin. Dyn., Psycho., and Life Sci., 19(1):1-12.

Kaplan, A., Fingelkurts, A., Fingelkurts, A., Borisov, B., and Darkhovsky, B. (2005). Nonstationary nature of the brain activity as revealed by eeg/emg: methodological, practical and conceptual challenges. Signal Processing, 85:2190-2212.

Laureys, S. and Tononi, G., editors (2009). Elsevier, Amsterdam.

Magrini, M., Virgillito, A., Barcaro, U., Bonfiglio, L., Pieri, G., Salvetti, O., and Carboncini, M. (2015). In Int. Workshop on Computational Intelligence for Multimedia Understanding (IWCIM 2015), Prague, 29-30 October 2015, IEEE Xplore Digital Library.

Monti, M., Vanhaudenhuyse, A., Coleman, M., Boly, M., Pickard, J., Tshibanda, L., Owen, A., and Laureys, S. (2010). New England Journal of Medicine, 362(7):579-589.
Navona, C., Barcaro, U., Bonanni, E., Martino, F. D., and Murri, M. M. L. (2002). Clinical Neurophysiology, 113(11):1826-1833.

Paradisi, P. and Allegrini, P. (2015). Chaos, Solitons \& Fractals, 81, Part B:451-462.

Paradisi, P., Allegrini, P., Gemignani, A., Laurino, M., Menicucci, D., and Piarulli, A. (2013). AIP Conference Proceedings, 1510:151-161.

Paradisi, P., Cesari, R., Contini, D., Donateo, A., and Palatella, L. (2009a). European Physical Journal: Special Topics, 174:207-218.

Paradisi, P., Cesari, R., Donateo, A., Contini, D., and Allegrini, P. (2012a). Nonlin. Processes Geophys., 19:113-126. Corrigendum, Nonlin. Processes Geophys., 19:685, 2012.

Paradisi, P., Cesari, R., Donateo, A., Contini, D., and Allegrini, P. (2012b). Reports on Mathematical Physics, 70:205-220.

Paradisi, P., Cesari, R., and Grigolini, P. (2009b). Central European Journal of Physics, 7:421-431.

Paradisi, P., Chiarugi, D., and Allegrini, P. (2015a). BMC Systems Biology, 9(suppl 3):s7.

Paradisi, P., Grigolini, P., Bianco, S., and Akin, O. (2008). International Journal of Bifurcation and Chaos, 18(9):2681-2691.

Paradisi, P., Kaniadakis, G., and Scarfone, A. (2015b). Chaos, Solitons \& Fractals, 81, Part B:407-411.

Paradisi, P., Kaniadakis, G., and Scarfone, A., editors (2015c). The emergence of self-organization in complex systems, volume 81, Part B of Chaos, Solitons \& Fractals. Elsevier, Amsterdam. Special Issue, Pages 407-588.

Peng, C.-K., Buldyrev, S., Havlin, S., Simons, M., Stanley, H., and Goldberger, A. (1994). Physical Review E, 49:1685-1689.

Plenz, D. and Thiagarjan, T. C. (2007). Trends in Neuroscience, 30:101-110.

Righi, M., Barcaro, U., Starita, A., Karakonstantaki, E., and Micheloyannis, S. (2008). Brain Topography, 21(1):43-51.

Robbie, J., Clarke, A., Barry, R., Dupuy, F., McCarthy, R., and Selikowitz, M. (2016). Clinical Neurophysiology, 127(5):2161-2166.

Solé, R. V. and Bascompte, J. (2006). Self-organization in Complex Ecosystems. Princeton University Press, Princeton.

Sornette, D. (2006). Critical Phenomena in Natural Sciences. Springer Series in Synergetics. SpringerVerlag, Berlin, 2 edition.

Spehar, B., Wong, S., van de Klundert, S., Lui, J., Clifford, C., and Taylor, R. (2015). Frontiers Human Neuroscience, 9(514).

Tononi, G. (2008). Biological Bulletin, 215:216-242.

Turalska, M., West, B., and Grigolini, P. (2011). Physical Review E, 83:061142.

Zueva, M. (2015). Frontiers in Aging Neuroscience, 7:135. 